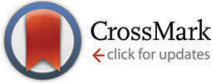

Cite this: Phys. Chem. Chem. Phys., 2015, 17, 20515

Received 28th May 2015, Accepted 22nd June 2015 DOI: $10.1039 / \mathrm{c} 5 \mathrm{cp} 03086 \mathrm{~b}$

www.rsc.org/pccp

\title{
Strong electron donation induced differential nonradiative decay pathways for para and meta GFP chromophore analogues $\dagger$
}

\author{
Tanmay Chatterjee, Mrinal Mandal, Venkatesh Gude, Partha Pratim Bag and \\ Prasun K. Mandal*
}

\begin{abstract}
$Z-E$ Isomerisation because of rotation around the exocyclic double bond (known as the $\tau$-twist) and not any other internal conversion has been reported to be the major nonradiative decay channel for nonhydroxylic unconstrained para and meta GFP chromophore analogues. The equation $\Phi_{f}+2 \Phi_{Z E}=1$ has been shown to hold well for both para and meta GFP chromophore analogues. If the above equation holds true, then upon reducing the extent of $Z-E$ isomerisation $\left(\Phi_{Z E}\right)$, the fluorescence quantum yield $\left(\Phi_{f}\right)$ should increase. To probe the above proposition two sets of non-hydroxylic unconstrained para and meta GFP chromophore analogues were synthesized. Quite interestingly by introducing the strongly electron donating $-\mathrm{NEt}_{2}$ group to the benzenic moiety these para and meta GFP chromophore analogues were shown to exhibit differential optical behaviour w.r.t. the extent of the solvatochromic shift, $\Phi_{f}, \Phi_{Z E}$, and $\tau_{f}$. For the first time it has been shown that the well accepted equation $\Phi_{f}+2 \Phi_{Z E}=1$ does not hold at all for these non-hydroxylic unconstrained meta analogues. Although $\Phi_{Z E}$ has been shown to be $<10 \%$, $\Phi_{\mathrm{f}}$ is much lower than the expected near unity value for these meta analogues. After detailed investigation into the nonradiative excited state decay channel, contrary to literature reports, energy gap law governed internal conversion and not $Z-E$ isomerisation was shown to be the major nonradiative decay channel for these meta analogues. Two models are put forward to understand the differential optical behaviour of these para and meta GFP chromophore analogues. Support from X-ray crystal structures, NMR experiments, and computational calculations has also been provided.
\end{abstract}

\section{Introduction}

$Z-E$ Isomerisation because of rotation around the exocyclic double bond ( $\tau$-twist, see Chart 1 ), and not any other internal conversion has been reported to be the major nonradiative decay channel, because of which the fluorescence quantum yield $\left(\Phi_{\mathrm{f}}\right)$ of the GFP chromophore ( $p$-HBDI, see Chart 1) in normal solvents is reduced. ${ }^{1-8}$ This proposition has been extended to other unconstrained GFP chromophore analogues. ${ }^{9-12}$ For example both $p$-HBDI and $p$-ABDI (Chart 1) have a $Z-E$ isomerisation quantum yield $\left(\Phi_{Z E}\right) \sim 0.5$ and $\Phi_{\mathrm{f}} \sim 0.0001 .^{9}$ However, meta analogues of these two molecules i.e. $m$-HBDI

Department of Chemical Sciences, Indian Institute of Science Education and Research (IISER), Mohanpur, Kolkata, West-Bengal, 741246, India.

E-mail: prasunchem@iiserkol.ac.in

$\dagger$ Electronic supplementary information (ESI) available: Experimental details, synthesis and characterization of GFP chromophore analogues, additional NMR data and stack plot, crystallographic table, additional steady state and time resolved spectroscopic data, etc. CCDC 1001355 and 1001357-1001359. For ESI and crystallographic data in CIF or other electronic format see DOI: 10.1039/ c5cp03086b
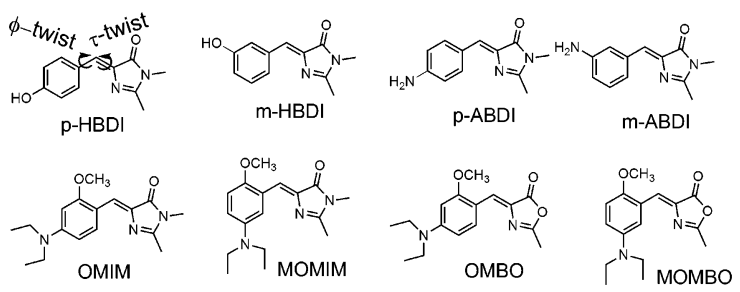

Chart 1 Chemical structures of the different GFP chromophore analogues.

and $m$-ABDI possess a $\Phi_{\mathrm{f}}$ of 0.0023 and 0.16 respectively. ${ }^{9-11}$ Their excited state fluorescence lifetimes $\left(\tau_{\mathrm{f}}\right)$ are also at least one order of magnitude higher than that of the para analogues. $^{9-11}$ Thus, enhancement of $\Phi_{\mathrm{f}}$ and $\tau_{\mathrm{f}}$ could be achieved by structural modification. It has been shown that the extent of charge transfer is much higher for the meta analogues in comparison to the para analogues. ${ }^{9,11}$ Thus, differential optical behaviour (w.r.t. $\Phi_{\mathrm{f}}$ and $\tau_{\mathrm{f}}$ values) has been shown for meta and para analogues. ${ }^{9-12}$ This difference has been explained on the basis of the "meta effect" in analogy with the behaviour observed for stilbene derivatives. ${ }^{10-18}$ Both for 
para and meta analogues in aprotic solvents it has been shown that ${ }^{9,11,12}$

$$
\Phi_{\mathrm{f}}+2 \Phi_{Z E}=1 \text {. }
$$

It has been reported that in aprotic solvents both for para and meta analogues $Z-E$ isomerisation is the major nonradiative decay channel. ${ }^{9-12}$ It has been reported that no other internal conversion plays any important role towards the non-radiative excited state decay channel. ${ }^{9,11,12}$ It has also been shown for constrained GFP chromophore analogues where $Z-E$ isomerisation is reduced that $\Phi_{\mathrm{f}}$ is enhanced. ${ }^{19-27}$ Thus, if the above eqn (1) holds true for unconstrained molecules, then, by structural modification, if the $\Phi_{Z E}$ value can be reduced then the $\Phi_{\mathrm{f}}$ value can be enhanced. In order to probe the above idea we synthesized two sets of para and meta analogues namely OMIM, OMBO (para analogues) and MOMIM, MOMBO (meta analogues) (see Chart 1). In all these molecules we introduced a strong electron donating diethylamino group with the belief that the stronger electron donating effect will suppress the $Z-E$ isomerisation and hence will improve the $\Phi_{\mathrm{f}}$ value. To avoid perturbation from H-bonding all the molecules and the solvents explored here are without hydroxyl groups.

\section{Results and discussion}

The extent of charge transfer reflected in the magnitude of solvatochromic shift has been observed to be much higher in meta analogues (141 nm for MOMIM and $176 \mathrm{~nm}$ for MOMBO from hexane to ACN) (see Fig. 1 for MOMIM and $\mathrm{ESI} \dagger$ for MOMBO) in comparison to their para analogues (OMIM $(\sim 30 \mathrm{~nm})$ and OMBO $(\sim 30 \mathrm{~nm})$ ). Similar observations for other meta analogues have been reported in literature. ${ }^{9,11}$ Also, $\Phi_{\mathrm{f}}$ of MOMIM is much higher than OMIM. Moreover the fluorescence lifetime $\left(\tau_{\mathrm{f}}\right)$ of the meta analogue MOMIM is $\sim 1000$ times higher (2.78 ns in ACN) than the para analogue OMIM (1.9 ps in ACN) (see Fig. 1). Similar observations have been observed for MOMBO and OMBO (see ESI $\dagger$ ). Thus, it is confirmed that the enhanced extent of charge transfer is reflected in high values of both $\Phi_{\mathrm{f}}$ and $\tau_{\mathrm{f}}$ in meta analogues in comparison to para analogues.

As a next step it was necessary to calculate the $\Phi_{Z E}$ values of all four analogues in different solvents. Interestingly, unlike literature reports, ${ }^{9,11}$ the para and meta analogues exhibit quite different $\Phi_{Z E}$ values (see Fig. 2, Table 1 and ESI $\dagger$ ). The $\Phi_{Z E}$ values for the para analogues (OMIM, OMBO) are $\sim 50 \%$, however, the meta analogues (MOMIM and MOMBO) exhibit $\Phi_{Z E}$ values of $\sim 10 \%$ or less in all solvents. No significant solvent dependence (from $\mathrm{CDCl}_{3}$ to $\mathrm{CD}_{3} \mathrm{CN}$, to DMSO- $\mathrm{d}_{6}$ ) was seen for either para or meta analogues. Thus, we can conclude that differential optical behaviour was observed between para and meta analogues (MOMIM and MOMBO) w.r.t. $\Phi_{\mathrm{f}}, \tau_{\mathrm{f}}$ as well as $\Phi_{Z E}$ values.

Moreover, as can be seen from Table 1, eqn (1) holds good for para analogues but the equation fails completely for the studied meta GFP chromophore analogues. Thus, an apparent correlation between reduced $\Phi_{Z E}$ and enhanced $\Phi_{\mathrm{f}}$ could be drawn for meta analogues. However, had $Z-E$ isomerisation been the major nonradiative decay channel for meta GFP chromophore analogues, its very small value $(<10 \%)$ would have increased the $\Phi_{\mathrm{f}}$ value to be close to unity. But this was not the case as $\Phi_{\mathrm{f}}$ for MOMIM was only $12 \%$ in hexane and $0.6 \%$ in ACN.
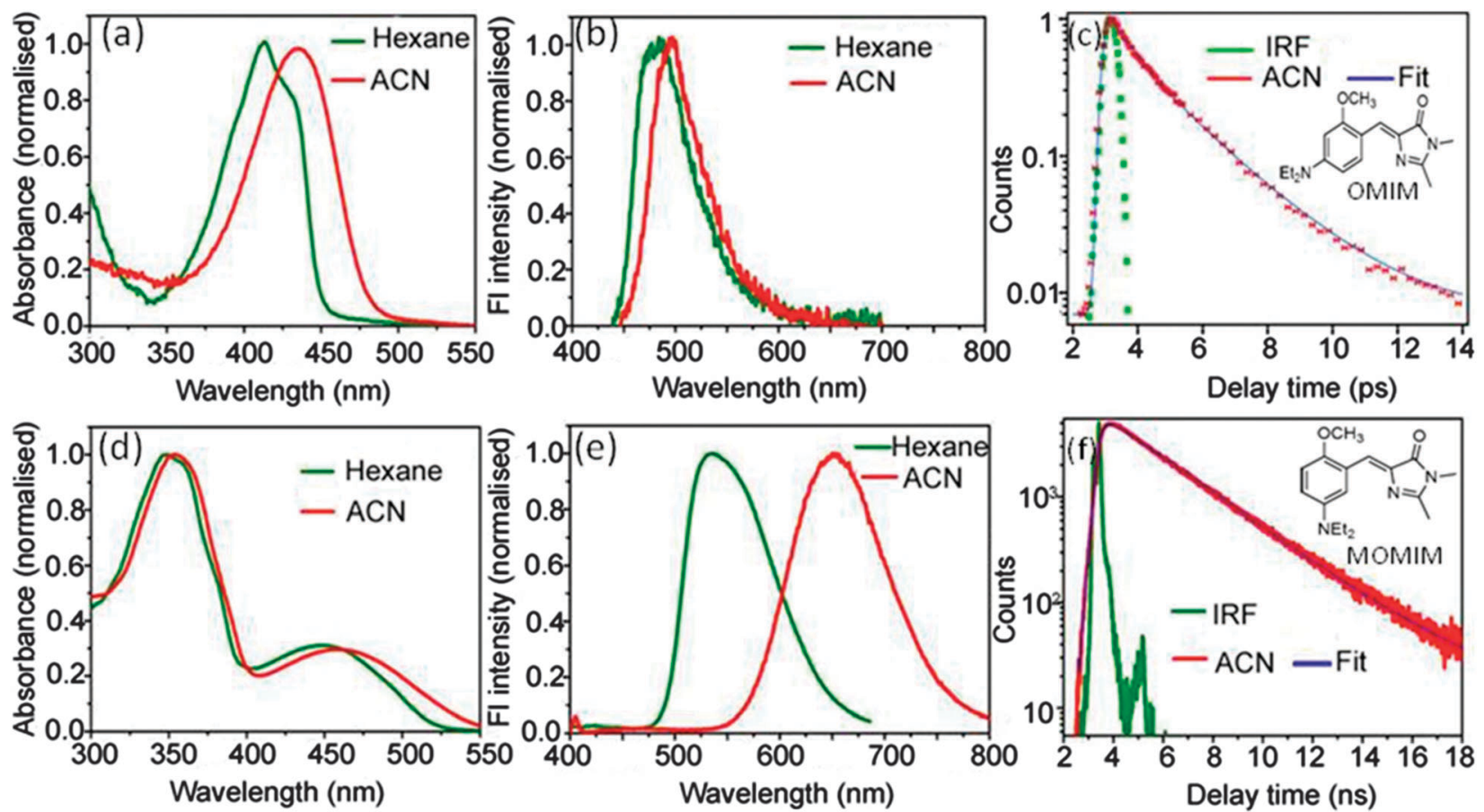

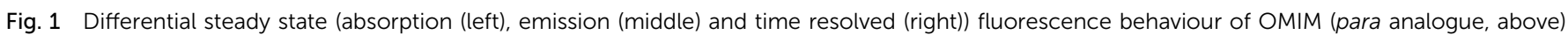
and MOMIM (meta analogue, below). 

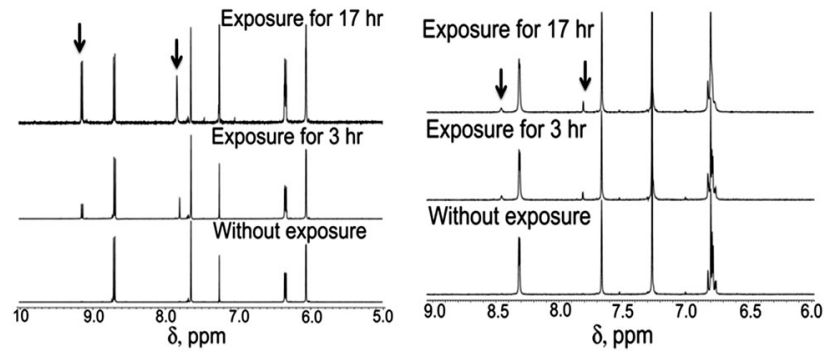

Fig. 2 Differential $Z-E$ isomerisation for OMIM (para analogue, left) and MOMIM (meta analogue, right).

Table 1 Experimental parameters related to $Z-E$ isomerisation

\begin{tabular}{|c|c|c|c|c|c|c|}
\hline Compound & Solvent & $\begin{array}{l}\% \text { of } \\
Z \text {-isomer }\end{array}$ & $\begin{array}{l}\% \text { of } \\
E \text {-isomer }\end{array}$ & $\Phi_{\mathrm{f}}$ & $\Phi_{Z E}$ & $\begin{array}{l}\Phi_{\mathrm{f}}+ \\
2 \Phi_{Z E}\end{array}$ \\
\hline \multirow[t]{3}{*}{ OMIM } & $\mathrm{CDCl}_{3}$ & 50 & 50 & $<10^{-3}$ & 0.48 & 0.9 \\
\hline & $\mathrm{CD}_{3} \mathrm{CN}$ & 46 & 54 & $<10^{-3}$ & 0.50 & 1.00 \\
\hline & DMSO-d $_{6}$ & 52 & 48 & $<10^{-3}$ & 0.52 & 1.04 \\
\hline \multirow[t]{3}{*}{ OMBO } & $\mathrm{CDCl}_{3}$ & 38 & 62 & $<10^{-3}$ & 0.60 & 1.2 \\
\hline & $\mathrm{CD}_{3} \mathrm{CN}$ & 48 & 52 & $<10^{-3}$ & 0.50 & 1.0 \\
\hline & DMSO-d $_{6}$ & 45 & 55 & $<10^{-3}$ & 0.55 & 1.10 \\
\hline \multirow{3}{*}{ MOMIM } & $\mathrm{CDCl}_{3}$ & NR & NR & 0.04 & 0.08 & 0.2 \\
\hline & $\mathrm{CD}_{3} \mathrm{CN}$ & NR & NR & 0.006 & 0.05 & 0.1 \\
\hline & DMSO- $\mathrm{d}_{6}$ & NR & NR & 0.006 & 0.04 & 0.0 \\
\hline \multirow{3}{*}{ МOMBO } & $\mathrm{CDCl}_{3}$ & NR & NR & 0.02 & 0.13 & 0.2 \\
\hline & $\mathrm{CD}_{3} \mathrm{CN}$ & NR & NR & 0.002 & 0.04 & 0.0 \\
\hline & DMSO-d $_{6}$ & NR & NR & 0.002 & 0.06 & 0.1 \\
\hline
\end{tabular}

${ }^{a}$ At the photo-stationary state (PSS). ${ }^{b}$ NR: PSS could not be reached even after $17 \mathrm{~h}$ indicating significantly slower kinetics for meta analogues in comparison to para analogues.

Thus, we can conclude that contrary to literature reports $Z-E$ isomerisation is not the major nonradiative decay channel for these meta analogues. Thus it is necessary to know what kind of non-radiative decay is involved in the photophysics of these meta analogues.

In order to understand why eqn (1) fails completely for these meta GFP chromophore analogues and also to know what kind of major nonradiative decay could be taking place, we carried out structural analysis starting from ground state to excited state. We obtained single crystal X-ray structures of all four compounds. These crystal structures are depicted in Fig. 3. As can be seen from Fig. 3 all four compounds remain in a planar $Z$ form with the-OMe group in the opposite direction to that of the imidazolidinone ring in the solid phase. As a next step, in order to know whether the same form persists in the solution phase, we carried out NMR measurements of all four compounds in different solvents. As can be seen from the NMR results (ESI $\dagger$ ) even in the solution phase the ground state remains in the $Z$ form. Thus, from single crystal X-ray and from NMR experiments we can conclude that the ground state structural form remains the same both in the solid and the solution phase for the meta analogues (and also for the para analogues). This means both meta and para analogues remain in same ground state structural form. Thus, we can make two important inferences: (i) that ground state structural heterogeneity does not exist; and (ii) that the differential optical behaviour is not due to ground state structural differences.

Ground state structural analysis could not provide significant clue regarding differential $\Phi_{Z E}$ values for para $(\sim 50 \%)$ and meta analogues $(<10 \%)$. Moreover, it is also not understood, why instead of having very low $\Phi_{Z E}$ values, the meta analogues have much lower (than unity) $\Phi_{\mathrm{f}}$. First, we tried to understand the reason behind the differential magnitude of $\Phi_{Z E}$ values for para $(50 \%)$ and meta $(<10 \%)$ analogues. Our experimental observation was that the extent of charge transfer (in the planar configuration) in the meta analogues is much higher than that of the para analogues. Computational calculation results (see Fig. 4)

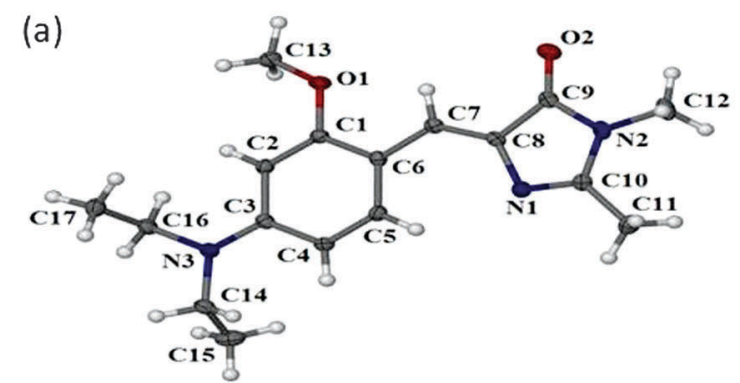

(c)

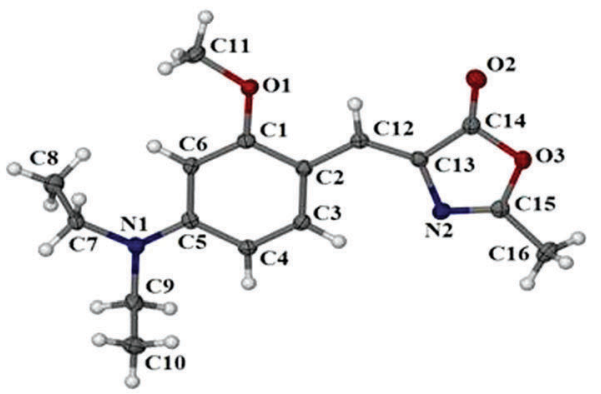

(b)

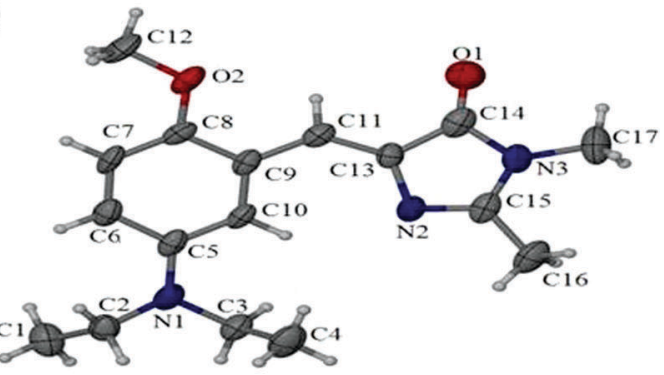

(d)

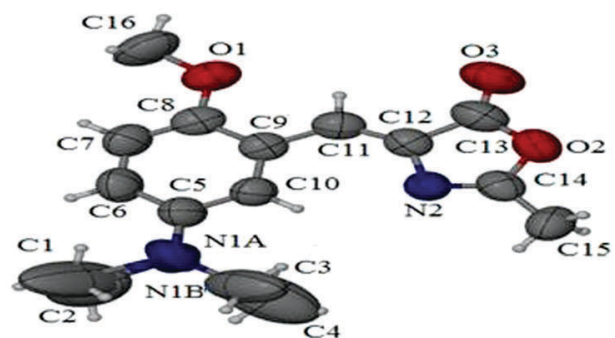

Fig. 3 Single crystal X-ray structure of $\operatorname{OMIM}(a), \operatorname{MOMIM}(b), \operatorname{OMBO}(\mathrm{c}), \operatorname{MOMBO}$ (d). 

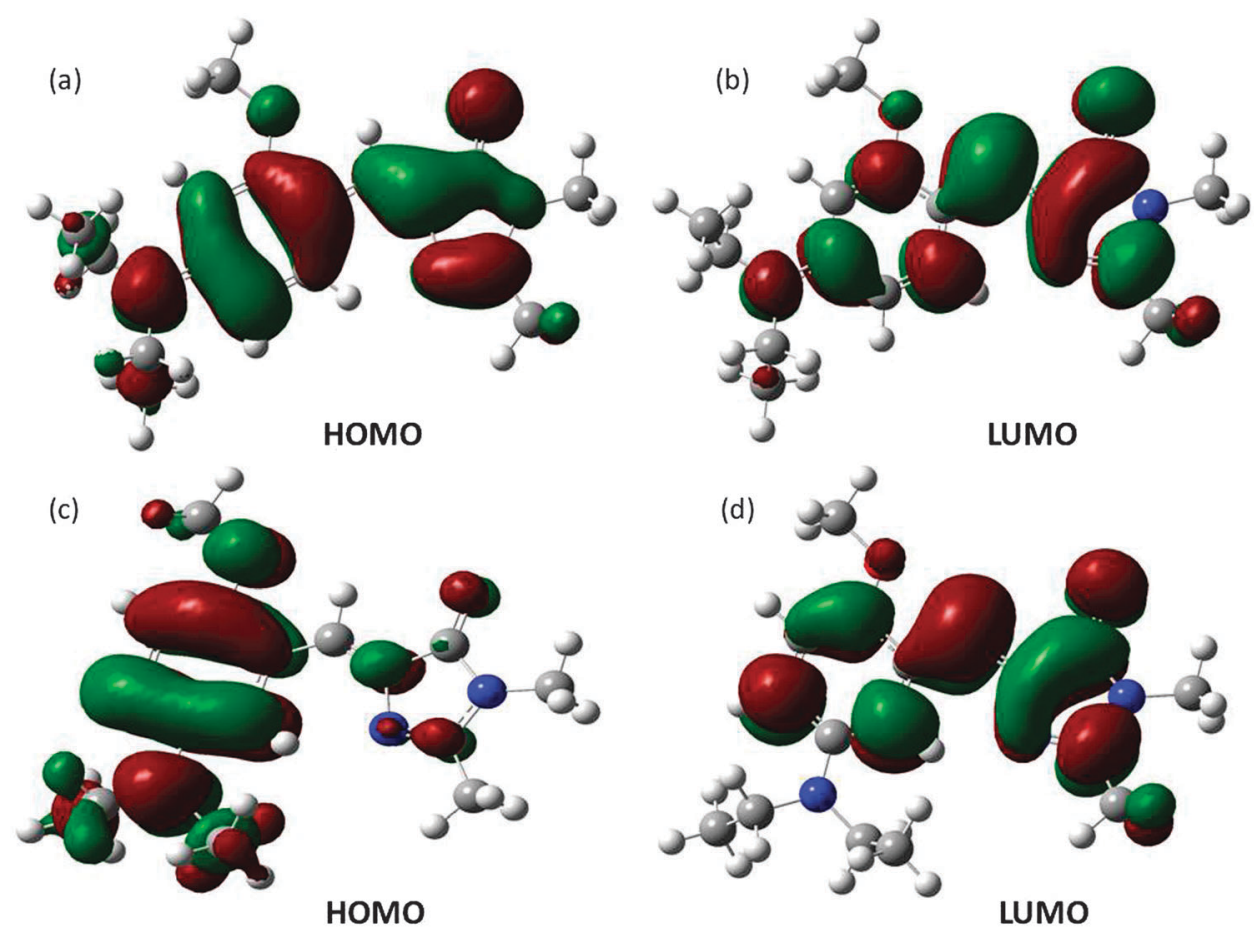

Fig. 4 Frontier molecular orbitals of OMIM ( $a$ and b) and MOMIM ( $c$ and d) calculated at the B3LYP/6-31G ${ }^{++}$level.

show that on going from the HOMO to the LUMO the extent of charge separation is much higher for meta analogues than para analogues. Hence, the emission spectra of the meta analogues should show bigger solvatochromic shifts than those of the para analogues. This is exactly what we obtained experimentally. Thus, the computational calculations support the experimental results.

After initial photo-excitation from the ground state $Z$ form to the FC excited state, the excited state species could either go to the planar ${ }^{1} \mathrm{~S}^{*}$ state or it could go to the $\tau$-twisted ${ }^{1} \mathrm{P}^{*}$ state. Which process will take place depends on the height of the (excited state) barrier between the planar and twisted state. Lowering the energy of the ${ }^{1} \mathrm{P}^{*}$ twisted state relative to the planar ${ }^{1} \mathrm{~S}^{*}$ state decreases the barrier height and vice versa. Applying a similar analogy to that proposed by Michl and Bonačić-Koutecký for simple alkenes, the ${ }^{1} \mathrm{P}^{*}$ state can be described as a combination of the biradical and charge transfer configuration. ${ }^{14,28,29}$

$$
\psi_{1 \mathrm{P}^{*}}=c_{1} \psi_{\text {biradical }\left(\mathrm{A}^{\bullet} \mathrm{B}^{\bullet}\right)}+c_{2} \psi_{\mathrm{CT}\left(\mathrm{A}^{+} \mathrm{B}^{-}\right)}+c_{3} \psi_{\mathrm{CT}\left(\mathrm{A}^{-} \mathrm{B}^{+}\right)}
$$

An electron donating para-substituent group stabilizes the twisted state through resonance and thereby decreases the energy of the twisted ${ }^{1} \mathrm{P}^{*}$ state. ${ }^{14}$ In stark contrast, a meta electron donating group due to very weak mesomeric interaction cannot stabilize the twisted state $\left({ }^{1} \mathrm{P}^{*}\right) .{ }^{14}$

The stabilization of the ${ }^{1} \mathrm{~S}^{*}$ state is much higher for meta analogues (as the polarity of the solvent increases), whereas according to the Michl and Bonačić-Koutecky model, ${ }^{28,29}$ stabilisation of the ${ }^{1} \mathrm{P}^{*}$ state is much higher for para analogues. Hence, for meta analogues the barrier height (from ${ }^{1} \mathrm{~S}^{*}$ to ${ }^{1} \mathrm{P}^{*}$ ) is much higher in comparison to para analogues (Scheme 1). Thus, meta analogues favour the planar rather than the twisted geometry in the excited state. This is why (solvatochromic) stabilisation of the planar ${ }^{1} \mathrm{~S}^{*}$ state is much higher for meta analogues. Moreover, because of the strong electron donating $-\mathrm{NEt}_{2}$ group (in comparison to the $-\mathrm{NH}_{2}$ group) the solvatochromic shift (from hexane to ACN) of MOMIM is much larger (142 nm) in comparison to $m$-ABDI (83 nm) (see ESI $\dagger$ ).

We have estimated the magnitude of stabilization of the charge transferred planar singlet state $\left({ }^{1} \mathrm{~S}^{*}\right)$ of the meta analogues MOMIM and MOMBO to be $11.9 \mathrm{kcal} \mathrm{mol}^{-1}$ and $14.45 \mathrm{kcal} \mathrm{mol}^{-1}$ in comparison to that of para analogues (OMIM and OMBO respectively) from steady state emission in $\mathrm{CHCl}_{3}$. The same for ${ }^{1} \mathrm{P}^{*}$ state was not possible to calculate due to the non-fluorescent character of the state.

However, unlike meta analogues, for para analogues (Scheme 1a) the ${ }^{1} \mathrm{P}^{*}$ state is energetically lower than the FC or ${ }^{1} \mathrm{~S}^{*}$ state. Thus, after initial excitation the molecule goes from the $\mathrm{FC}$ or ${ }^{1} \mathrm{~S}^{*}$ state to the ${ }^{1} \mathrm{P}^{*}$ state for para analogues. Thus, the $\Phi_{Z E}$ value is much higher for para analogues (OMIM and OMBO) $\left(\Phi_{Z E} \sim 50 \%\right)$. From the ${ }^{1} \mathrm{P}^{*}$ state the molecule relaxes to the ground electronic state in an ultrafast nonradiative fashion resulting in a much smaller $\Phi_{\mathrm{f}}$ value ( $\sim 0.0001$ ) as well as $\tau_{\mathrm{f}}$ value (femto-picosecond) for para analogues. Whereas for meta analogues, since the energy of the ${ }^{1} \mathrm{P}^{*}$ state is comparable to the FC state or (much) higher than the ${ }^{1} \mathrm{~S}^{*}$ state, there is less probability that the meta analogues go from the ${ }^{1} \mathrm{~S}^{*}$ state to the ${ }^{1} \mathrm{P}^{*}$ state. Hence, the $\Phi_{Z E}$ value is much reduced $\left(\Phi_{Z E}<10 \%\right)$ for meta analogues (MOMIM and MOMBO). Thus, for meta analogues, molecule decays from ${ }^{1} \mathrm{~S}^{*}$ state, significantly radiatively resulting in much higher $\Phi_{\mathrm{f}}$ value $(\sim 0.12$ in hexane) as well as high $\tau_{\mathrm{f}}$ value (5.22 ns in hexane).

Schemes somewhat similar to Scheme 1 have been reported previously. ${ }^{9,11}$ However, that model can't explain the experimental 
(a)

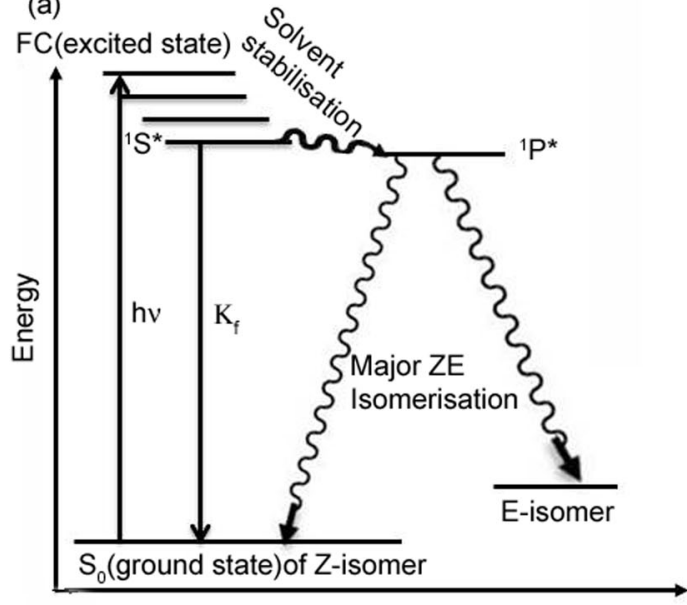

(b)

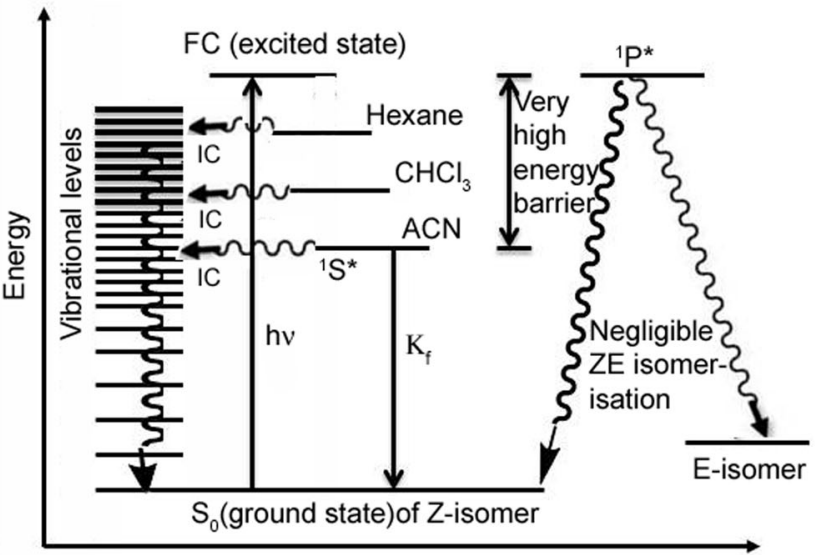

Scheme 1 Photophysical processes of (a) para analogues (OMIM and OMBO) and (b) meta analogues (MOMIM and MOMBO). (Drawn in accordance with the Perrin-Jablonski diagram shown in ref. 30.)

results we obtained. Moreover, though the twisted ${ }^{1} \mathrm{P}^{*}$ state was conjectured to be much higher in energy than that of the planar ${ }^{1} \mathrm{~S}^{*}$ state in $m$-ABDI, still much higher $Z-E$ isomerisation $\left(\Phi_{Z E}=0.45\right)$ in aprotic solvents has been reported. ${ }^{11}$ As $\Phi_{\mathrm{f}}+2 \Phi_{Z E}=1$ holds for both $m$-ABDI and $p$-ABDI in aprotic solvents, it was concluded that $Z-E$ isomerisation is the only nonradiative decay channel available to both meta and para compounds in spite of the slow isomerisation kinetics of the former. ${ }^{9,11,12}$ Similar result was noted for $m$-HBDI by Tolbert et al. in nonaqueous solvents. ${ }^{10}$

Since the two sets of para and meta analogues exhibit widely different $\Phi_{Z E}$ values, quite contrary to previously reported results, $Z-E$ isomerisation is not the major nonradiative decay channel for meta analogues in aprotic solvents and thus the reported models could not explain our experimental results. Thus, two different models need to be provided to understand the differential optical behaviour for para and meta analogues. These differential observations have been outlined in Scheme 1. From the scheme we could understand the electronic reason for such a reduced $\Phi_{Z E}$ value for meta analogues in comparison to the high $\Phi_{Z E}$ values for para analogues.

However, from $\Phi_{\mathrm{f}}+2 \Phi_{Z E}=1$, as the $\Phi_{Z E}$ value is $<10 \%$, the $\Phi_{\mathrm{f}}$ value should have been close to $80 \%$ for the meta analogues. But the experimental observation says that the $\Phi_{\mathrm{f}}$ value is at best $12 \%$ (in hexane). This means there exists another non-radiative decay channel for meta analogues. Thus, it is necessary to know what kind of nonradiative decay channels could possibly be the reason for deviation of $\Phi_{\mathrm{f}}$ from its near unity value for the meta analogues. Because of enhanced charge transfer, the emission maximum of MOMIM is considerably red shifted (547 $\mathrm{nm}$ in hexane and $688 \mathrm{~nm}$ in $\mathrm{ACN}$ ) in comparison to the other meta analogues (e.g. $m$-ABDI has an emission maximum of $495 \mathrm{~nm}$ in hexane and $578 \mathrm{~nm}$ in ACN) reported in the literature. ${ }^{9,11}$ The emission maxima of MOMIM and MOMBO are in the red whereas those for OMIM or OMBO as well as other reported $m e t a$ analogues ( $m$-HBDI and $m$-ABDI) are in the green region. Thus, the excited states of MOMIM and MOMBO are much more energetically stabilized in comparison to OMIM, OMBO and
$m$-HBDI and $m$-ABDI. Thus, the energy difference between the stabilized excited state and the FC ground state is much smaller in case of MOMIM and MOMBO. Thus, following the energy gap law, nonradiative emission i.e. internal conversion from the stabilized excited state to the FC ground state becomes highly significant in the cases of MOMIM and MOMBO. ${ }^{31-35}$

However, the same is not so significant for para analogues or other meta analogues like $m$-ABDI. ${ }^{9,11,12}$ Thus, because of the facile energy gap law governed internal conversion, which is a nonradiative decay process, the $\Phi_{\mathrm{f}}$ value deviates greatly from the expected $80 \%$ value. As the polarity of the solvent increases the energy gap between the stabilized/solvated excited state and ground state decreases and hence the nonradiative internal conversion becomes more facile. This also explains why $\Phi_{\mathrm{f}}$ decreases with increasing polarity in the case of MOMIM (0.12 in hexane to 0.006 in ACN) and MOMBO (0.10 in hexane to 0.002 in ACN). On going from hexane to acetonitrile, the radiative decay rate constant $\left(K_{\mathrm{f}}=\Phi_{\mathrm{f}} / \tau_{\mathrm{f}}\right)$ decreases from $0.21 \times 10^{8} \mathrm{~s}^{-1}$ to $0.021 \times 10^{8} \mathrm{~s}^{-1}$ and the nonradiative decay rate constant $\left(K_{\mathrm{nr}}=\left(1-\Phi_{\mathrm{f}}\right) / \tau_{\mathrm{f}}\right)$ increases from $1.74 \times 10^{8} \mathrm{~s}^{-1}$ to $3.57 \times 10^{8} \mathrm{~s}^{-1}$, respectively, for MOMIM. Thus, the large extent of the charge transfer (strong 'meta effect') induced barrier in twisting makes $Z-E$ isomerisation a minor nonradiative decay pathway for MOMIM and MOMBO. Rather, it is the energy gap law governed facile internal conversion which becomes the major nonradiative decay pathway for MOMIM and MOMBO. This observation is in complete contrast to that of the para analogues and the reported meta analogues ( $m$-ABDI and $m$-HBDI) for which $Z-E$ isomerisation is the only major nonradiative decay channel and for which any other internal conversion has been reported to be not at all important in aprotic solvents.

\section{Conclusions}

In conclusion, we synthesized two sets of non-hydroxylic unconstrained para and meta GFP chromophore analogues which exhibited differential optical properties in aprotic solvents w.r.t. $\Phi_{\mathrm{f}}$, 
$\Phi_{Z E}$, and $\tau_{\mathrm{f}}$ values. For the meta analogues because of the strong "meta effect" the extent of charge transfer was much greater in comparison to the para analogues. $\Phi_{\mathrm{f}}$, and $\tau_{\mathrm{f}}$ values of the meta analogues are 1000 times higher than those of the para analogues. $\Phi_{Z E}$ values for para analogues are $\sim 50 \%$ and for meta analogues are $<10 \%$. The well accepted equation $\Phi_{\mathrm{f}}+2 \Phi_{Z E}=1$ holds good for para analogues but fails completely for meta analogues. In contrast to existing literature reports, $Z-E$ isomerization has been shown not to be the major nonradiative decay channel for meta analogues. Rather, because of enhanced charge transfer and hence a reduced energy gap between the stabilised excited state and ground state, the energy gap law governed facile internal conversion was shown to be the major nonradiative decay channel for meta analogues. These observations for meta analogues are in stark contrast to the existing literature of unconstrained non-hydroxylic GFP chromophore analogues. Two models have been provided which successfully explain the differential optical behaviour of para and meta GFP chromophore analogues. Thus, by structural variation the extent of charge transfer can be modulated and thus the nature of optical behaviour as well as the pathway of nonradiative excited state decay could be controlled.

\section{Experimental details}

\section{Calculation of $Z-E$ isomerisation efficiency}

To measure the $Z-E$ isomerisation quantum yield $\left(\Phi_{Z E}\right)$, solutions of low (mM) concentration range were used and irradiated with $370 \mathrm{~nm}$ light (8 W mercury lamp) without applying any special filter. The irradiation was followed by ${ }^{1} \mathrm{H}$ NMR at different time intervals. Since distinctive ${ }^{1} \mathrm{H}$ NMR signals are obtained for $Z$ and $E$ isomers, integration of the NMR peak corresponding to each isomer allowed us to know the isomer ratio at a certain time of irradiation. $p$-HBDI served as the reference standard $\left(\Phi_{Z E}=0.48\right.$ in $\mathrm{ACN}) .{ }^{9}$ The isomerisation quantum yield was calculated with the following equation: ${ }^{9,36}$

$$
\frac{C_{\mathrm{r}} \times V_{\mathrm{r}} \times P_{\mathrm{r}}}{\Phi_{Z E \mathrm{r}} \times t_{\mathrm{r}}}=\frac{C_{\mathrm{s}} \times V_{\mathrm{s}} \times P_{\mathrm{s}}}{\Phi_{Z E \mathrm{~s}} \times t_{\mathrm{s}}}
$$

where $C$ is the concentration of the substrate, $P$ is the amount (\%) of the initial $Z$-isomer that undergoes conversion to the $E$-isomer after irradiation, $V$ is the volume of the solution, $t$ is the irradiation time, and the subscripts $r$ and $s$ stand for the reference standard ( $p$-HBDI) and the substrate (OMIM, OMBO, MOMIM and MOMBO) respectively. $P$ was determined from the integrated intensity of the ${ }^{1} \mathrm{H}$ NMR peak for the $E$-isomer. The sample of $\sim 10^{-3} \mathrm{M}$ concentration was prepared in an NMR tube (Sigma Aldrich) and kept in a UV chamber for irradiation with a $370 \mathrm{~nm}$ light source. The back isomerisation in the ground state was also monitored by NMR. It was observed that back isomerisation after exposure was possible in the presence of light for OMIM and OMBO but not for MOMIM or MOMBO. However, in the dark the ground state back isomerisation from the $E$-isomer to the initial $Z$-isomer did not happen over a duration of three days for either of the derivatives. So, to keep ground state back isomerisation out of the calculation of $\Phi_{Z E}$, the NMR tube was wrapped with aluminium foil after removal from the irradiation chamber. For the $\Phi_{Z E}$ value calculation, the sample was irradiated for a short time so that the irradiated sample containing a mixture of $E$ and $Z$ isomers was far from reaching a photo-stationary state (PSS).

\section{Abbreviations used}

Acronyms like $p$-HBDI, $m$-HBDI, $p$-ABDI, $m$-ABDI have previously been used in the literature. , $^{90}$

GFP green fluorescence protein

OMIM $\quad o$-methoxy imidazolidinone

OMBO $o$-methoxybenzoxazolidinone

MOMIM meta diethylamino o-methoxy imidazolidinone

MOMBO meta diethylamino $o$-methoxy benzoxazolidinone

\section{Conflicts of interest}

The authors declare no competing financial interests.

\section{Acknowledgements}

PKM thanks IISER-Kolkata for financial help and instrumental facilities. Support from the Fast-Track Project (SR/FT/CS-52/2011) of DST-India is gratefully acknowledged. PKM acknowledges Prof. P. Ramamurthy (NCUFP) for his help in femtosecond decay measurements. TC, and PPB thank CSIR, MM and VG thank IISER-Kolkata for their respective Fellowship.

\section{References}

1 W. Weber, V. Helms, J. A. McCammon and P. W. Langhoff, Proc. Natl. Acad. Sci. U. S. A., 1999, 96, 6177-6182.

2 A. Follenius-Wund, M. Bourotte, M. Schmitt, F. Iyice, H. Lami, J. J. Bourguignon, J. Haiech and C. Pigault, Biophys. J., 2003, 85, 1839-1850.

3 M. E. Martin, F. Negri and M. Olivucci, J. Am. Chem. Soc., 2004, 126, 5452-5464.

4 P. Altoe, F. Bernardi, M. Garavelli, G. Orlandi and F. Negri, J. Am. Chem. Soc., 2005, 127, 3952-3963.

5 S. Olsen and S. C. Smith, J. Am. Chem. Soc., 2007, 129, 2054-2065.

6 S. A. Olsen, J. Chem. Theory Comput., 2010, 6, 1089-1103.

7 G. J. Huang and J. S. Yang, Chem. - Asian J., 2010, 5, 2075-2085.

8 A. Baldridge, S. R. Samanta, N. Jayaraj, V. Ramamurthy and L. M. Tolbert, J. Am. Chem. Soc., 2010, 132, 1498-1499.

9 J.-S. Yang, G.-J. Huang, Y.-H. Liu and S.-M. Peng, Chem. Commun., 2008, 1344-1346.

10 J. Dong, K. M. Solntsev, O. Poizat and L. M. Tolbert, J. Am. Chem. Soc., 2007, 129, 10084-10085.

11 C. W. Cheng, G. J. Huang, H. Y. Hsu, C. Prabhakar, Y. P. Lee, E. W. G. Diau and J. S. Yang, J. Phys. Chem. B, 2013, 117, 2705-2716.

12 G. J. Huang, J. H. Ho, C. Prabhakar, Y. H. Liu, S. M. Peng and J. S. Yang, Org. Lett., 2012, 14, 5034-5037. 
13 E. M. Crompton and F. D. Lewis, Photochem. Photobiol. Sci., 2004, 3, 660-668.

14 F. D. Lewis, S. R. Kalgutkar and J. S. Yang, J. Am. Chem. Soc., 1999, 121, 12045-12053.

15 H. K. Sinha and K. Yates, J. Am. Chem. Soc., 1991, 113, 6062-6067.

16 H. E. Zimmerman, J. Am. Chem. Soc., 1995, 117, 8988-8991.

17 H. E. Zimmerman, J. Phys. Chem. A, 1998, 102, 5616-5621.

18 K. M. Solntsev, O. Poizat, J. Dong, J. Rehault, Y. Lou, C. Burda and L. M. Tolbert, J. Phys. Chem. B, 2008, 112, 2700-2711.

19 L. Wu and K. Burgess, J. Am. Chem. Soc., 2008, 130, 4089-4096.

20 A. Baldridge, K. M. Solntsev, C. Song, T. Tanioka, J. Kowalik, K. Hardcastle and L. M. Tolbert, Chem. Commun., 2010, 46, 5686-5688.

21 M. S. Baranov, K. A. Lukyanov, A. O. Borissova, J. Shamir, D. Kosenkov, L. V. Slipchenko, L. M. Tolbert, I. V. Yampolsky and K. M. Solntsev, J. Am. Chem. Soc., 2012, 134, 6025-6032.

22 C. C. Hsieh, P. T. Chou, C. W. Shih, W. T. Chuang, M. W. Chung, J. Lee and T. Joo, J. Am. Chem. Soc., 2011, 133, 2932-2943.

23 K. P. Kent and S. G. Boxer, J. Am. Chem. Soc., 2011, 133, 4046-4052.

24 K. Do and S. G. Boxer, J. Am. Chem. Soc., 2011, 133, 18078-18081.
25 A. Baldridge, S. Feng, Y. T. Chang and L. M. Tolbert, ACS Comb. Sci., 2011, 13, 214-217.

26 J. S. Paige, K. Y. Wu and S. R. Jaffrey, Science, 2011, 333, 642-646.

27 T. Chatterjee, D. Roy, A. Das, A. Ghosh, P. P. Bag and P. K. Mandal, RSC Adv., 2013, 3, 24021-24024.

28 V. Bonačić-Koutecký, J. Köhler and J. Michl, Chem. Phys. Lett., 1984, 104, 440-443.

29 V. Bonačić-Koutecký and J. Michl, J. Am. Chem. Soc., 1985, 107, 1765-1766.

30 B. Valeur, Molecular Fluorescence - Principles and Applications, WILEY-VCH Verlag GmbH, Weinheim, 2002.

31 N. J. Turro, V. Ramamurthy and J. C. Scaiano, Modern molecular photochemistry of organic molecules, University Science Books, Sausalito, CA, 2010.

32 R. Englman and J. Jortner, Mol. Phys., 1970, 18, 145-164.

33 C. Liu, K. C. Tang, H. Zhang, H. A. Pan, J. Hua, B. Li and P. T. Chou, J. Phys. Chem. A, 2012, 116, 12339-16345.

34 J. V. Caspar and T. J. Meyer, J. Phys. Chem., 1983, 87, 952-957.

35 M. Bixon and J. Jortner, J. Phys. Chem., 1994, 98, 1289-1294.

36 G. J. Huang, C. W. Cheng, H. Y. Hsu, C. Prabhakar, Y. P. Lee, G. E. W. Diau and J. S. Yang, J. Phys. Chem. B, 2013, 117, 2695-2704. 